\title{
10. REGISTER
}

\subsection{Ortsregister}

Aachen $470^{532}, 484664$

Abbéville $430^{164}$

Acqui $233^{426}$

Agde $465^{491}, 467^{508}, 476^{582}$

Agen $446^{313}, 478^{606}$

Agrigent $463^{474}$

Aire-sur-l'Adour 483652

Aix-en-Provence $156^{+105}, 449^{347}, 488$, $490^{720}$

Alba Julia 469523

Albano 104, 448, 453

Albi $80,125 f ., 437^{234}, 438^{234}, 442^{278}$, $449347,463,48,+652$

Alès $449^{347}, 468^{523}$

Algesiras $151^{86}, 227^{404}, 425,490^{720}$

Amiens $140^{36}, 434^{200}, 453,465,478,479^{606}$

Ancona, Mark 248, 273, 276, 298, 439f., 443

Andalusien 425

Andorra 466

Angers $86,480^{629}, 481^{629}, 490^{720}$

Angoulême $490^{720}$

Anjou $147^{69}$

Annonay $485,486^{680}$

Antivari 311

Antoing 148

Apt $487^{694}, 490^{720}$

Aquileia 232, 311, 431 $174,458^{424}$

Aquitanien $177^{+191}, 178-184,424,445$

Aragon $24^{36}, 37,51,133 f$., 136, 244, 273598, $287,425,431,436,439,466,470,474$, $477,481^{632}, 489^{+716}$

Arezzo $428,495^{757}$

Arles 467508

Armagh 487

Arras 49, 80, 94f., 199, 424, 430 484664

Assisi $426,480^{+621}$

Asti $233,236^{432}$

Astorga $172,436^{215}, 482^{645}$

Aubenas 479

Auch $447^{329}, 483^{652}$

Autun $426^{133}, 438^{234}, 477^{598}, 484^{664}, 485$

Auxerre $80,436^{203}, 446^{313}, 447^{+329}, 487$

Aversa 169153

Avignon 11, 30, 47, 49f., 52, 55, 59, 62, $72^{130}, 99,103,108,133,150,152$,
162-164, 166, 174f., 180, 184f., 192-196, 198, 200, 202, 205+305, 209, 211f., $213^{340}$, $215,220^{369}, 222 \mathrm{f}$., 233f., 236, 239, 245f., $247^{480}, 250^{491}, 251,258 f$., 261f., $271^{587}$, $272,275^{+605}, 276 f$., 280-284, 290, 299 , $301,303^{709}, 305^{+720}, 306 \mathrm{f} ., 308^{731}, 310$, $312,323^{12}, 327-329,411^{69}, 414^{83}, 425^{+124}$, $426,428,432,436 f$., $440^{+263}, 441,444$, $446,447+329,452,454,456,458,460 \mathrm{f}$., $463-465,467^{+491}, 469,472,476-479$, $482-485,487,489-493$

Avila $427^{133}, 429,431$

Avranches 149

Babylon 212, 214, 221, 222

Bamberg $457^{415}$

Barcelona $436^{+215}, 459^{436}, 462,463^{474}$, $467^{508}, 475^{+581}$

Bari $4311^{174}$

Barjols 472

Bayeux $458^{424}, 460,465^{491}, 483652,485^{+676}$, $490^{720}$

Beaulieu-sur-Ménoire 465

Beauvais 435,484664

Bellapaise 446

Belpech (Aude) 124, 463, 465

Benevent 276, 425 $118,431^{174}, 439$

Bergamo $458^{424}$

Bergerac 447329

Berlin 9

Besançon $437^{234}, 438^{234}$

Besset $435^{203}$

Beyssac (Corrèze) 449

Béziers $46,447^{329}, 470^{532}$

Billom $485^{676}$

Bologna $9,34,51,81^{26}, 248,311,425$, $426^{+130}, 428,439,443-445,451,457,468$, 480

Bordeaux 282, $425^{118}, 446^{313}, 477$

Bouchet 462

Boulbonne $463^{+465}$

Boulogne 160

Bourges $429^{156}, 447^{329}, 449^{346}$

Brabant $160,449^{346}$

Braga $427^{133}$

Brantôme 424

Bratislava $311,472^{546}$ 
Bremen $484^{664}, 492^{739}$

Brescia 311

Bretagne 149, 160, 166, 196, 199

Brétigny 450

Brive $455,475,490^{720}$

Brügge $426^{164}, 435^{+206}, 436^{215}, 462^{456}$

Burgos $459^{436}, 482^{645}, 490^{720}$

Cahors $436^{215}, 442^{278}, 444^{291}, 453 f ., 468^{523}$, 471

Calais $52,141^{+38}, 209^{+324}, 299,327^{37}$

Cambrai $449^{346}, 468^{+523}, 473^{555}, 483^{652}$

Cambridge 223384

Campagna und Marittima 248, 298, 304, $428,431,439$

Campines 495

Campion $476^{586}$

Canterbury $166,169^{+155}, 171,173,189,455$

Carcassonne $454,465,470^{532}, 492^{739}$

Carpentras $425^{118}, 470^{532}, 479606,481^{629}$

Carretret 438

Carthagena 481

Cassel $140,476^{586}$

Cassino 466

Castelnau-Montratier (Lot) 442

Castelnaudary 449346

Caumont 464

Cavaillon $444^{291}, 473^{555}, 479^{606}, 487^{694}$

Cesano 286

Chalons-sur-Marne $458^{424}$

Chalons-sur-Saône $437^{234}, 438^{234}, 478^{606}$

Chartres $80,199,277,279,428,429+158$, 456,480

Châtelus (Limousin) 428

Cherbourg 195

Clairvaux 85

Clémency $480^{629}$

Clermont $80,438^{234}, 449^{+346}, 462^{456}$, $478^{606}, 484^{664}, 485^{676}$

Cognac $465^{49}, 476^{582}$

Coimbra 459

Colombier 483f.

Conches $449^{347}$

Condom $447^{329}$

Condroz $478^{606}$

Corneto 426

Corrèze 455

Crécy $141,195,201,202^{292}, 203,210^{324}$, 326

Cuenca 425

Dacien 267

Daumar $455^{399}$
Dax $444^{291}$

Déaulx (Gard) 438

Dijon $468^{523}$

Dinant 149

Dôle 468

Douai $148,435^{206}$

Elna 459436

Elne $476^{586}, 478^{606}$

Ely $452^{370}$

Embrun $80,273,438,462^{456}, 480$

England 14, 37, 52, 133, 143-145, 147, 151, $152^{90}, 153,157,160-162,164-166,169$ $171,174,179 f$., $183 f ., 186,190,191^{242}$, 193f., 199280, 207f., $212^{332}, 222,229 f$., $233,327,424,433,448-450,452 \mathrm{f} ., 455$, $461,477,481,488,491,494$

Esplechin 144, $151^{87}$

Essones 456

Esztergom $312,468^{523}, 472^{546}$

Étampes $431^{173}$

Évreux $457^{415}, 469^{523}, 474$

Exeter 188

Exminster 188

Eyrein (Corrèze) 468

Faenza 266

Fécamp 47, 49f.

Ferrara 232, 248, 311, 428, $479^{606}$

Fiesole $470^{532}$

Flandern 140, 183, 197271, 199, 481

Florenz 23, 284, 428, 476, 495+757

Foix 434,466

Fontfroide 463

Forlì 23

Frankfurt $493^{739}$

Frankreich 14, 37, 52, 133, 143-145, 151, $152^{90}, 153,157,160 f ., 164-166,174,176$, 178, 181f., 193, 197, 202 $293,207,209$, $212^{332}, 222,229 f$., $319,321,327,429,433$, 448-450, 452f., 461, 473, 477, 481, 488, 491f., 494

Fréjus 472

Gap $449^{347}, 465^{491}, 484^{664}, 492^{739}$

Gascogne 199, 482

Genf $437^{234}, 444^{291}, 465^{491}, 473^{555}$

Gent $426^{164}$

Genua $232,275,276^{+607}, 287,303^{707}, 310 f$., $431^{174}$

Gerona $463^{474}, 470^{532}, 472^{546}, 490^{720}$

Gibraltar 229, 425

Grado $232,431^{174}$ 
Granada $151^{86}$

Grandselve 463

Grenoble 479606

Groningen $476^{582}$

Guarda $481^{629}$

Guyenne 50, 140f., 195, 198f., 426164, 447

Hamburg $24^{38}, 476^{582}$

Hemingbrough $207^{312}$

Hennegau 144, 481

Hildesheim $457^{415}$

Huesca $426^{133}, 434^{200}, 436^{215}$

Imola 266

Irland 165,453

Israel 227

Italien $14,227,247,288,425 f$., 428,439 , $443,491,496$

Játiva 489

Jerez 450

Jerusalem $80,212-215,222,323^{12}, 446$

Kastilien $229,435,462,466,468$

Kerpen $476^{582}$

Klosterneuburg 86

Koblenz 493739

Köln $461^{456}, 462^{456}, 476^{582}, 479606,487^{694}$, $492^{739}$

Konstantinopel 318,459431

Korsika 248, 428

Krakau 86

La Chaise-Dieu $60,66,117^{152}, 122,466$, 468f., $471^{+540}, 478$

Lagrasse 46,465

Langres $437^{234}, 438^{234}, 449^{346}, 457^{415}$, $476^{582}, 484664$

Languedoc 453,475

Lanta 474

Laon $456,483,484^{664}$

Laudun $490^{720}$

Laurac (Aude) $449^{346}$

Lavaur $478^{606}$

Le Mans $465^{491}, 477^{598}$

Le Pouget 444

Le Puy $483,484^{664}, 485^{676}$

Lectoure $447^{329}$

Leicester $168^{150}, 495$

Lens $435^{203}$

León $425,427^{133}, 435^{215}, 460^{437}, 490^{720}$

Lérida $435^{215}, 462,470^{532}$

Lézat 467
Lichfield $482^{645}$

Limoges $80,428,435^{215}, 447,455,465,471$, $478 f$., 487

Limousin 424

Lincoln $164^{+135}, 167^{142}, 170,171^{+163}, 172$, $173^{173}, 174^{176}, 175,185,451^{364}, 474$, $476^{586}, 482^{645}, 496$

Lisieux $201,205^{305}, 424^{+118}, 487$

Lissabon $469^{523}, 482^{645}$

Lombardei $131^{1}, 133^{5}, 134,231,240$, $273^{598}, 311,464$

Lombez $23,436,457$

London $167^{147}, 186$

Lucca 284

Luçon $442^{278}, 483^{652}$

Lüttich $20^{15}, 22^{22}, 36,59^{73}, 133^{6}, 152$, $273^{598}, 440^{263}, 441,449^{347}, 462^{456}, 467^{507}$, $470^{532}, 478^{606}, 483^{652}, 495$

Lyon $9,18,80,437^{+234}, 438^{234}, 441,460$, $484^{664}, 492^{739}$

Maastricht $462^{456}, 476^{582}, 487^{694}$

Mâcon $438^{234}$

Maguelonne $436215,440^{263}, 467^{508}, 470^{532}$, $473^{+555}, 478^{606}, 492^{+729}, 493^{739}$

Mailand 232f., 236432, 240, 287, 300, 311, $424,428,431^{174}, 443^{286}, 467$

Mainz 383, 434200, 436, 447, 458 $424,462^{456}$, $476^{+586}, 487^{694}, 493^{739}$

Malestroit $52,149,151^{+87}, 161-163,165$, $178,195 f ., 303^{709}, 327^{37}, 433,488$

Mallorca 51, 133f., $136^{20}, 273^{598}, 431,452$, 489

Manfredonia $308^{729}$

Marseille $458^{424}, 472^{546}$

Maumont 45, 469

Mende 491, $492^{739}$

Metz 448

Minden 469523

Mirepoix $434^{200}, 463^{474}$

Modena 232, 300,311, 443

Mondonedo $469^{523}$

Mons $476^{586}$

Montauban 436215

Montault $484,486^{680}$

Montefiascone 282, 477

Montfavet 441f.

Montmirail $469^{523}$

Montolieu 467

Montolivet $470^{532}, 471$

Montpellier 438f., 441, 466, 471, 475, 485, 492

Montpézat 488f. 
Moussy-le-Neuf 487

München 86

Nabinaux 445

Narbonne $46,451,470^{532}, 478^{606}$

Narni 248, 298

Nassington 495

Navarra 452

Neapel 14, 23, 37, 52, 108f., 133-135, 233, 244-247, 251-254, 256-259, 268, 270, $271^{+587}, 273-276,277^{+613}, 278-284,286$, $288,291-293,298,301^{+701}, 302,307-312$, $323,326-328,426,428 f$., 431-433, 438f., $446,447^{329}, 448,466,480^{620}, 495$

Negroponte $459^{431}$

Nevers $94,431^{173}, 483,484^{664}, 485$

Nicosia $445,446^{313}, 447^{329}$

Nîmes $125,425^{118}, 463,465^{491}, 470^{532}$, 479606

Nizza $495^{760}$

Nocera $476^{582}$

Normandie 195, 197, 199, 319

Norwich $164,175,184,468^{517}$

Noyon $449^{+347}, 458^{424}, 478^{606}$

Olmütz 86

Oloron $434^{200}$

Oppeln 434200

Orense 427133

Orléans $97,442^{278}, 468,469^{523}, 478^{+606}$, 485

Orvieto $276,440^{263}$

Ostia $30,129^{193}, 443,450,484^{+661}$

Oxford $426^{133}$

Padua 274, 277, 279, 311f., 317, 461 ${ }^{+448}$, 495

Palencia $459^{436}, 482^{645}, 484^{664}$

Palestrina 182, 488, 492

Pamiers $80,434+200$

Pamplona $4720^{32}$

Paris 9, 11, 49f., 53, 63f., 93, 97, 141, 145, $149,152,195,244,384,431 f ., 445^{+304}$, 446, $449^{346}, 455 f$., $457^{415}$, 463f., $468 f$., $475,477 f ., 479^{+613}, 480,485,487,489^{708}$ 490

Parma 232, 311, 443

Pavia $213^{340}, 311$

Périgueux $446^{313}, 447^{329}, 448,455$

Perpignan 431, 436

Perugia $248,298,440^{263}, 443,477^{+592}$, $482^{645}, 490,495^{757}$

Piacenza 232
Piemont 464

Pisa $428,431^{174}, 443,495^{757}$

Pistoia 284

Poitiers $142,435^{215}, 465^{491}, 473^{555}, 477$, $479^{606}, 483^{652}, 490^{720}$

Polen 467510

Pontorson 149

Ponthieu 478

Porto 460, 474

Portugal 435,462

Posen $132^{2}$

Prag 283, 434200, 444, $462^{456}$

Prato $458^{424}$

Provence 246, 260, 282, 479, 480620, 495760

Psalmody $446^{313}$

Ragusa 311

Ravenna 172, 247, 428, 431174, 459436

Reggio Emilia 232, 311, 443

Reims $317^{770}, 460,461^{456}, 462^{456}, 465^{491}$

Rennes 468

Richmond $207^{312}$

Rieti $248,260^{539}, 298$

Rieux 467508

Riez $488,492^{739}$

Rimini 430,459

Rochester $152^{89}, 208^{318}$

Rodez $435^{+215}, 436^{215}, 437,440,465^{491}$, $478^{606}, 490$

Rom 9, 18, 23, 55, 214, 233, 249f., 260, 270 , $282,283^{+636}, 284^{+638}, 288,291,298$, $300^{+698}, 302^{+707}, 303,305-309,312,326 f$., $383,431-433,437,439 f$., 458, 461, 466, $476^{586}, 477,479,484,491 \mathrm{f}$.

Romagna 80, 247f., 251, 266, 293f., 298, $300,327,426,428,443$

Romans 439

Rosiers d'Egletons (Corrèze) $45 f$., 490

Rouen $46,49 f ., 468 f$., $478^{606}, 484^{664}, 490^{720}$

Rouvignac 449346

Sabina $104,167,284,294,440,481,482^{645}$

Saint-Baudil 47

Saint-Bertrand-de-Comminges $446^{313}$, 469523

Saint-Denis $456^{+410}$

Saint-Exupéry 465

Saint-Félix-de-Caraman 472

Saint-Flour $492^{730}$

Saint-Germain-les-Belles 471

Saint-Jean-d'Angély 469

Saint-Malo $483^{652}$

Saint-Omer $\mathbf{4 7 6}^{586}$ 
Saint-Pantaléon de Lapleau 47

Saint-Papoul $425^{118}, 479^{606}$

Saint-Paul-Trois-Châteaux $454,468^{523}$

Saint-Pons-de-Thomières $470^{532}$

Saint-Quentin 483

Saintes $446^{313}, 451^{364}, 465^{491}, 478^{606}$

Salamanca $426^{133}, 436^{215}$

Salisbury $440^{263}, 451^{364}, 495^{+756}$

Salzburg $131^{1}, 311$

San Gimignano 282

San Giorgio in Liri 308

Sangatte $141^{38}$

Santiago de Compostela $469^{523}, 482^{645}$

Sardinien 248,428

Saulieu $438^{234}$

Saverdun $467^{508}$

Savigny 47

Schottland $165,179,453$

Segovia $469^{523}$

Senlis $435^{203}, 487$

Sens $49,66,85,116,424,431^{173}$

Sevilla $435^{215}, 449^{347}, 470^{532}, 482^{645}$

Siebenbürgen $468^{523}$

Siena $276,277^{613}, 495^{757}$

Sisteron $438^{234}, 478^{606}, 490^{720}$

Sizilien, Königreich 158, 244, 254, 256f., $264,266,271,273 \mathrm{f}$., $275^{603}, 284^{638}, 298$, $318,424,439,459$

Sluys 141

Smyrna $53,227^{+404}$

Soest $492^{739}$

Soissons $457^{415}, 461^{456}, 479^{06}, 484^{664}$

Sorgues $432 f$., 482

Spanien $227,425,462 f ., 473,477$

Split 311

Spoleto, Dukat 248, 280, 298, 428, 439f., $495^{757}$

Stebenheth $168^{151}$

Straßburg 382

Stregnäs $492^{739}$

Tarbes $446^{313}, 447^{329}$

Tarentaise $438^{234}$

Tarifa $425 f$.

Tarragona $435^{215}, 459^{436}, 490^{720}$

Terni 248, 298

Terra di Lavoro $301^{704}$

Thérouanne $424,431^{173}, 457^{415}, 461^{456}$, $462^{456}$

Todi $248,298,469^{523}, 495^{757}$

Toledo $80,425,426^{+133}, 442^{278}, 449^{347}$, $451^{364}, 481,495$

Tortosa $434^{200}, 465^{491}, 470^{532}, 490^{720}$
Toscana 484661

Toulon $425^{118}$

Toulouse $80,81^{27}, 425^{+118}, 434^{196}$, $447-449,451^{+364}, 454 f$., $463^{474}, 466$, $467^{508}, 468^{523}, 469^{523}, 474,475^{+576}$, $476^{583}, 483^{652}, 488,490^{720}, 492^{+739}$

Tournai $80,148,203^{296}, 430,431^{173}, 435^{203}$, $461^{456}, 462^{456}$

Tours $86,458^{424}, 472,473^{555}$

Tourtoirac $446^{313}$

Treviso $311,312,316$

Trier $455^{404}, 462^{456}, 476,487^{694}, 493^{739}$

Troyes $431^{173}, 437^{234}$

Tudela 462

Tulle $46,80,465,469 f$.

Turin 310

Tusculum 123f., 126, 128f., 156, 168, 182 , $244,432^{180}, 477$

Tuszien $244,248,250^{491}, 252,256,264,298$

Ungarn $23,131^{1}, 224,278-280,282,286 f$., 298f., 301, 307-312, 318f., 460

Uppsala $492^{739}$

Urbino 443

Urgel $79 f ., 466,476,477^{592}$

Utrecht 476

Uzès $440^{263}, 451^{363}, 462^{456}, 470^{532}, 490^{720}$

Västeras $492^{739}$

Vaison-la-Romaine $470^{532}, 484664$

Valence $472^{546}$

Valencia $437^{234}, 459^{436}, 470^{532}, 471^{532}, 473$, $477^{598}, 482^{645}, 489,490^{720}$

Vannes 149

Varennes $438^{234}$

Vatikan 86

Vauclaire 448

Venaissin, Comtat 56,194257, 492

Venedig 37, 53, 232, 287, 311, 428, 464

Ventimiglia $275,276^{607}$

Vercelli 233

Verdun $462^{456}, 476^{586}$

Verona $240,287^{651}, 311$

Vienne $425^{118}, 474,484^{664}$

Villemur 434

Villeneuve-lès-Avignon $38,448,470$

Vincennes 49, 383, 486

Viterbo $426,466,492$

Viviers $437^{234}, 438^{234}, 444^{291}, 457^{416}$, $465^{491}, 469^{523}, 479606,480^{629}, 490^{720}$

Westminster 187,190

Wilton $207^{312}$ 
10. Register

Winchester 189

Wimbledon 189

Wistow $207^{312}$

Worms 484664

Xanten $457^{415}, 487^{694}$

York 188f., 192, $194^{255}, 207^{+312}, 424,447^{329}$

Ypern 144
Zamora $426^{133}, 469^{523}$

Zara 311

Zaragossa $46,80,426^{133}, 436^{215}, 465^{+491}$, $470^{532}, 482^{645}$

Zypern 53, 445 


\subsection{Personenregister}

Da Clemens VI./Kardinal Pierre Roger auf nahezu jeder Seite genannt wird, wurde auf die Aufnahme seines Namens in das Personenregister verzichtet.

Aaron, Bruder des Mose $155,156^{+107}$

Absalom 264

Adhémar Robert, Kardinal $23,77^{7}, 80$, $81^{26}, 82^{30}, 120^{163}, 170,185,188,191-194$, 207f., 41483, 424, 429

Agnes, Schwester von Élie Talleyrand de Périgord 271, 272+591, 446

Agnes Blannbekin $35^{87}$

Agnès Emperière, Mutter von Kardinal Pierre Bertrand sen. 485

Aimeric de Châtelus, Kardinal $70^{121}, 77^{66}$. $80,81,82^{30}, 100^{95}, 120^{163}, 133^{5}, 135,138$, $235^{430}, 241^{454}, 244,246$ f., $248^{485}$, $250-252,253+501,254^{+507}, 255-257$, $258^{529}, 259^{+531}, 260^{+539}, 261^{+544}$ $262^{+549}, 266-269,270,275,299,326$, $414^{83}, 424,428 f$., $444,454^{396}, 464$

Albertus della Scala $250^{492}$

Albornoz, Gil, Kardinal $78^{10}, 80-82$, $134^{12}, 283,425-427,445,450,482$, $484^{661}, 485^{667}$

Alexander III., Papst $30^{63}$

Alfons XI., König von Kastilien $227^{404}$, $425^{+124}, 435$

Almodie Roger, Schwester Clemens' VI. 45,478

Alvarus Pelagius $35^{92}, 482$

Ambrosius, Kirchenvater $67^{105}, 68^{111}, 96$, $116^{149}, 123,293$

Amiano de la Mothe, Vater von Kardinal Galhard 451

Andreas Ghini Malpigli, Kardinal $37,77^{7}$, $78^{12}, 79,80,82^{30}, 93,133^{5}, 134,429-431$, 436

Andreas von Ungarn 23, 33, 52, $169^{153}$, 245f., 254, 270-272, 274, 275 ${ }^{+606}, 276^{609}$, 277f., $280,302^{706}, 308,326,439,444,447$, $452,460,467,474^{571}$

Androuin de la Roche $152^{90}, 426$

Anglic Grimoard 39

Annibaldo Ceccano, Kardinal 23, 31, $38^{+104}, 44,87^{43}, 120^{163}, 133^{+5}, 138,142$, 145f., $147^{+69}, 148$, 155f., $163,165 f$., $167^{145}, 168,170,175,178,194^{255}, 195^{+261}$, 196f., 204, 206+310, 207 312 , 211f., 215 , $222,298,299^{+686}, 300 f ., 302^{+707}, 303^{+709}$, $304^{+716}, 305-307,308^{+729}, 309^{+733}, 311$,
$312^{750}, 326 f ., 414^{83}, 431-433,441^{271}, 444$, $449,488,494^{750}$

Anonimo Romano, Chronist $38^{104}, 285$, 300, 304-308, 440

Antonius, Heiliger 461

Apollinaris Sidonius 293

Aristoteles $67,116^{149}, 242,268,313^{+757}$, $317+772,328^{42}$

Armandus de Bellovisu $442^{280}$

Arnaud II., Vicomte de Caraman 472

Arnaud de Pellagrue $458^{424}$

Arnaud de Via, Kardinal 444

Arnaud de Villemur, Kardinal $78^{10}, 80$, $81^{26}, 434$

Astorge Dufort 300

Augustinus, Kirchenvater $67 \mathrm{f} ., 70^{121}, 96$, $212,213^{+342}, 226$

Augustinus Triumphus 35,77

Balduin, Erzbischof von Trier 447, 476

Baldus de Ubaldis, Kanonist 490

Baluze, Étienne 6, 472 542

Barnabas, Apostel 222

Barthélemy de Colombier, Vater von Kardinal Pierre Bertrand jun. 483

Bartholachio de Magnardo 251

Bartholomäus de Burgassh 189

Bartolomeo di Jacopo da Valmontone $285^{646}$

Bartolomeo da Urbino 66

Batseba, Frau Urijas 264

Beattie, Blake $489^{712}$

Beda Venerabilis 226

Benedikt XII., Papst 32f., 50f., 5767, 58, $77,144,151^{87}, 193,243^{464}, 273,435 \mathrm{f}$., $439,441,444^{291}, 455^{+403}, 456,459+433$, $460,463^{+466}, 467,474,476,480,482 \mathrm{f}$.

Benoît II. de Jean, Vater von Kardinal Gaucelme 453

Berard II. von Ceccano 431

Berardus von Orvieto 223384

Berlière, Ursmer 3

Bernard d'Albi, Kardinal 23, 2436, 30f., $120^{163}, 133^{5}, 136^{20}, 138,220^{369}, 327^{39}$, $414^{83}, 434-437$

Bernard de Caulason $452^{370}$

Bernard Délicieux 474 
Bernard de la Tour, Kardinal $77^{6}, 77^{7}, 82$, $120^{163}, 414^{83}, 437 \mathrm{f}$.

Bernarda de Jean, Mutter von Kardinal Gaucelme 453

Bernhard von Clairvaux 66, 69, 85f., 89, $106^{118}, 116 \mathrm{f}$., $118^{+156}, 158^{115}, 212$, 238f., $241,265-267,270,296,317^{770}, 318$, $411^{69}$

Bertrand de Baux $277^{+616}, 279+626$

Bertrand de Deux, Kardinal $27^{54}, 31^{+69}$, $104,108-110,120,133^{5} ; 138,158^{114}, 163$, 165f., 175, 184215, 237 $436,246,270$, $273^{+596}, 274^{+599}, 275,276^{+609}, 277^{+613}$ $278,280-286,287+655,288,292-296$, $297^{+682}, 299,301^{703}, 307,310,326^{+30}$, $328^{41}, 438-440,444,467,491$

Bertrand Jobelin 149

Bertrand de Montfavès, Kardinal $77^{4}$, 441f., 481

Bertrand du Pouget, Kardinal 23, 30, 3173, $73^{136}, 78^{11}, 120^{163}, 219^{363}, 223-228$, $246^{479}, 324,414^{83}, 433^{193}, 439,442-445$, $453^{379}, 468,473^{550}$

Bertrande Roger 45, 46

Bertrandus Atgerius, Kardinal $31^{71}$

Bertrandus de Cornelio $77^{3}$

Bertrandus Paravicini, Bischof von Bologna 480

Billanovich, Giuseppe $285^{646}$

Birgitta von Schweden 11, 307, 321, 329, $432^{181}, 491^{726}$

Blanche de Bourbon, Gemahlin Peters von Kastilien 450, 462, 468

Boccaccio, Giovanni 247

Boethius 317

Bonifaz VIII., Papst 11, 54, 303, 431, 459

Brunessende von Foix 446

Buridan 47

Caecilia, Heilige 126

Caillet, Louis 4

Caleph 221

Calixt II., Papst 329

Calmette, Joseph 140

Cassiodor $211^{331}$

Catherine de Courtenay, Herzogin von Tarent 274600

Catherine de Lagarde, Schwester von Kardinal Gérard de Daumar 455

Catull 291663

Christus 225f., 228f., 267, 271 $586,325^{25}$

Cicero 291663

Clemens IV., Papst 244
Clemens V., Papst 6, 438, 447320, 473, 493

Cola di Rienzo $52,55,282^{+635}, 283^{+636}$, $284^{+638}, 285 f$., $292-294,300^{+698}, 301^{703}$, 302f., 307, 326, 440, 458, 461, 495

Colonna, Aloisia, Mutter von Kardinal Niccold Capocci 476

Colonna, Giovanni, Kardinal $32^{77}, 120^{163}$, $414^{83}, 432,457,458$

Colonna, Pietro, Kardinal $32^{77}, 476$

Colonna, Stefano, Vater von Kardinal Giovanni Colonna 457

Cornelius Nepos $291^{663}$

Daniel, Prophet $116,155^{99}$

David, Prophet $100^{95}, 262^{549}, 264^{+557}, 265$, 293, 294, 3214

Denzel, Markus A. 5

Dominikus, Heiliger 68

Dotz, Johannes 47

Duchesne, François $424^{112}, 425^{119}, 451^{363}$, $455^{403}, 456^{408}, 467,470,489^{710}$

Durandus von S. Pourçain 433

Edward II., König von England 183, 453

Edward III., König von England 49f., $78^{16}, 140 f ., 144,147^{+72}, 148^{+76}, 149-151$, 160, 163f., 167-172, 175-177, 180-184, $186,190,192-194,195^{+261}, 196-198,200$, $201^{289}, 202,204-208,310,327^{37}, 441$, 455, 477, 494750

Elias, Prophet 221

Élie de Nabinal, Kardinal $77^{7}, 80,81^{23}$, $82^{30}, 83^{31}, 445,446$

Élie Talleyrand de Périgord, Kardinal $21^{20}, 31^{+68}, 33,37,39,42,43^{123}, 68,104$, $108-110,192^{+249}, 195^{261}, 253,254^{508}, 271$, $272^{+594}, 310,313,323^{18}, 430^{+168}$ $446-449,454^{396}, 460,462,469,474^{+571}$, $477^{+596}, 485^{667}$

Élise de Got, Nichte Clemens' V. 451

Élise Roger, Nichte Clemens' VI. 437

Ernst von Pardubitz, Erzbischof von Prag 283

Esau, Bruder Jakobs 108

Esra, Prophet $212^{332}, 214,216$

Étienne Aubert, Kardinal (Papst Innocenz VI.) $6,28^{56}, 30,32,37,77^{7}, 80 f$., $82^{31}$, $120^{163}, 133^{5}, 195^{+261}$, 196f., 206f., 211f., $215,222,254^{508}, 283,299,326,414^{83}$, 425f., 429, 433+193, $440,444^{294}, 448$, 449-452, 461f., 464, 467f., $471^{+540}, 487$, 490,492

Étienne de la Baume-Montrevel 441 
Étienne de la Garde, Bruder von Kardinal Gérard de Daumar $455^{398}$

Eugen III., Papst 21, 85f., 89, 116

Fauval de Vaudencourt 149

Fiorenzo Capocci, Vater von Kardinal Niccolò Capocci 476

Francesco Odonis 147

Francesco Ordelaffi $23,251^{+496}, 424$

François de Baux 466

François de Meyronnes 47

Franziskus von Assisi, Heiliger $486^{686}$

Friedrich, König von Aragon 439

Froissart, Jean, Chronist 141, $196^{264}$

Galhard de la Mothe, Kardinal $24^{38}, 37$, $120^{163}, 168,173,246^{479}, 414^{83}, 436$, $442^{278}, 444,451 f$., $454^{396}$

Gaston, Graf von Foix 438

Gaucelme de Jean, Kardinal $31^{+74}, 37$, $120^{163}, 138,163,165 f ., 168^{+151}, 175$, $207^{312}, 414^{83}, 444,450^{351}, 453 \mathrm{f}$.

Gaucerande de l'Isle-Jourdain 457

Gérard de Daumar, Kardinal 39, 44, 777, $80,81^{23}, 82^{30}, 192^{+245}, 455 f$., 475

Gérard de Ventadour 301

Géraud d'Aigrefeuille $465^{488}$

Gilles li Muisis $430^{+167}$

Gilles Rigaud, Kardinal $24^{38}, 78^{10}, 80$, $81^{23}, 456 \mathrm{f}$.

Giovanni Bernier de Fayt 67

Giovanni di Luca 305

Giovanni Regina $313^{755}$

Giovanni de Vico 284, 294, 440

Goliath 264

Gozzo da Rimini, Kardinal $24,120^{163}$, $414^{83}, 454,459 f$.

Gratian, Kanonist 54

Gregor der Große, Kirchenvater $68^{111}, 69$, $91^{62}, 107,116,117^{152}, 123,124^{174}, 153$, 161,323

Gregor VII., Papst 18

Gregor IX., Papst 23

Gregor X., Papst 18, 34

Gregor XI., Papst, vgl. Pierre Roger de Beaufort

Gui de Boulogne, Kardinal 2336, 30+67, 31f., $37,43^{123}, 77^{7}, 80,81^{27}, 82,83^{31}$, $120^{163}, 131^{1}, 133^{5}, 138^{+29}, 152^{90}, 273^{598}$, $277^{614}, 298 f$., $301^{704}, 303,306^{725}, 309$, $310^{+738}, 311-314,317-319,323^{18}, 414^{83}$, $433^{193}, 448^{+334}, 460-463,485^{667}, 487^{695}$, $494^{751}$
Guido von Parma 308

Guillaume d'Aigrefeuille, Kardinal $24^{36}$, $61^{79}, 77^{6}, 78^{10}, 80,82,83^{31}, 229^{410}, 465 f$., $471^{534}$

Guillaume Ami, Bischof von Apt 144

Guillaume d'Aure, Kardinal $27^{54}, 36,37$, $120^{163}, 129,246^{479}, 273^{598}, 414^{83}, 444$, $454,467,492^{729}$

Guillaume Balbet 149

Guillaume de Beaufort, Vater von Kardinal Pierre Roger de Beaufort 490

Guillaume de Besse, Vater von Kardinal Nicolas de Besse 478

Guillaume Court, Kardinal $21^{20}, 24^{38}$, $27^{54}, 31,37,120^{163}, 123-129,133^{5}, 134$, 138, 231-233, 235f., 238-240, 241454, $242-244,247,249^{487}, 250,267^{570}, 275$, $296,299,321^{4}, 326,328^{43}, 414^{83}$, $463-465,480^{619}$

Guillaume Fournier, Bruder Benedikts XII. $436^{229}$

Guillaume de la Garde, Bruder von Kardinal Gérard de Daumar $455^{398}$

Guillaume Grimoard 462, 492

Guillaume de la Jugée, Kardinal 2438-40, $45,61^{79}, 74,77^{61}$. $81^{27}, 87-89,97,120^{163}$, $229410,414^{83}, 468 f$., $471^{534}$

Guillaume Lamy, Bischof von Chartres $205^{308}, 480$

Guillaume de Mandagout, Kardinal 438

Guillaume Roger, Bruder Clemens' VI. $45,46,52^{45}, 455,491^{728}$

Guillaume Roger, Neffe Clemens' VI. 472

Guillaume de la Tour, Neffe von Kardinal Bernard de la Tour 436

Guillelmus de Bos 162

Guillelmus Duranti $131^{2}$

Guillemain, Bernard 2, 4f.

Guillemette de Mestre, Mutter Clemens' VI. 45,469

Guillemette Roger, Mutter von Kardinal Guillaume de la Jugée 468

Guy de Robert, Erzbischof von Chartres $428^{149}$

Hefele, Carl Joseph 1

Heinrich, Graf von Lancaster $189^{236}$

Heinrich, Erzbischof von Sens, gen. "Sanglier« 116

Heinrich von Virneburg, Erzbischof von Mainz 436, 447, 476

Heinrich von Kalkar 47

Henry Knighton, Chronist 151 
Hergemöller, Bernd-Ulrich 12

Hieronymus, Kirchenvater $67,68^{111}$, $116^{149}, 159,291,293,317$

Hiob, Prophet 216, 226

Hoberg, Hermann 5

Höfler, Constantin 321

Hostiensis, Kanonist 19

Hrabanus Maurus 226, 295

Hugh Neville 171, 175, 184+216

Hugues de Baux $313^{755}$

Hugues Géraud, Bischof von Pamiers 443, $453^{+379}, 488$

Hugues de Pommart 149

Hugues Roger, Kardinal 24, 45f., $61^{79}, 77^{6}$, $77^{7}, 80,81^{23}, 120^{163}, 229^{410}, 277^{614}, 310$, $414^{83}, 469-471$

Humbert II., Dauphin von Vienne 163, $261,429,436,439,475$

Ildebrandinus Conti, Bischof von Padua $274,285^{646}$

Imbert Dupuis, Kardinal $43^{123}, 121^{163}$, $414^{83}, 444,471 f$.

Innocenz III., Papst $292^{667}$

Innocenz VI., Papst, vgl. Étienne Aubert

Isabella, Königin von England 140, 186, $450,463^{472}$

Isidor von Sevilla $66^{102}, 72^{130}, 156^{104}$

Ivo, Heiliger (Yves Hélory de Tréguier) $21^{21}, 383,424,452,482$

Jacob von Artevelde 141

Jacobus von Savoyen 234427

Jacopo Stefaneschi 25, 136, 431, 433

Jacques de Besse 46

Jacques de la Jugée, Vater von Kardinal Guillaume de la Jugée 45, 468

Jakob, Bruder Esaus 108

Jayme II. von Mallorca 436, 439

Jean II., König von Frankreich, gen. »der Gute « $46,142,197,272^{592}, 313,456^{412}$, $460 f$.

Jean III., Herzog von Brabant 148

Jean, Graf von Armagnac 438

Jean d'Annonay $137^{23}$

Jean le Bel, Chronist $142,210^{324}$

Jean Birel, Kartäuserprior 448

Jean de Caramain, Kardinal $78^{10}, 80,472 \mathrm{f}$.

Jean de Cardaillac $28^{56}, 51^{43}, 77^{3}, 449^{348}$, $473^{551}$

Jean de Comminges, Kardinal 30,33, $120^{163}, 163,175,207^{312}, 272,277^{614}, 310$, $414^{83}, 442^{278}, 444,473 f$.
Jean Dupin 58

Jean Hessekint 384

Jean des Moulins, Kardinal $78^{10}, 80,81^{23}$, $82^{31}, 475 f$

Jean Quidort $36^{94}$

Jean de Roquetaillade $21^{20}, 42,57,448$, $450,464^{+484}$

Jean de Vienne 435

Jeanne de Boulogne 313

Jeanne de Bourgogne, Königin von

Frankreich 205

Jeremias, Prophet 116

Jesus, vgl. Christus

Jetro, Schwiegervater des Mose 19

Joab, at. Heerführer 293

Johann von Böhmen $201^{290}, 443 f$.

Johann von Verden $78^{17}$

Johanna von Burgund, Königin von Frankreich 485

Johanna I., Königin von Neapel 22, 52, $224,233,240,244,245^{+473}, 246$ f., 252 , $253^{+504}, 254,256-259,260^{533}, 268$, $271,276^{+607}, 277-279$, 281f., 286, 301f., $308,310 f ., 428^{150}, 429,439,444,460$, 471

Johannes, Apostel $108^{126}$

Johannes der Täufer $155^{100}$

Johannes XXII., Papst 11, 32f., 47, 49, 77 , $129,156^{105}, 186^{226}, 193,206^{310}, 408^{58}$, $428,431^{178}, 432 \mathrm{f}$., $434,441^{+271}, 442^{+281}$, $443,445,451-453,457,460,463,469$, 471f., $473550,474,481,483$, 485f., $488^{+702}, 493$

Johannes Paul II., Papst $143^{44}$

Johannes Caballinus de Cerronibus $28 \mathrm{f}$.

Johannes de Gravina, Herzog von Durazzo 271, 446

Johannes de Gres 163

Johannes de Manfredis 251

Johannes Paleologos, Kaiser von Byzanz 479

Johannes Parisiensis $35^{92}$

Johannes de Ripparia 273, 276

Johannes Schadland 489

Johannes de Thoresby 184

John de Grandisson, Bischof von Exeter $190^{239}$

John Offord $164,175,185$

John of Shoreditch 192

Joseph, Heiliger 88, 269

Josephus, Historiograph 290

Josua, Nachfolger des Mose $221^{+372}$ 
Karl IV., röm.-dt. Kaiser 22, 52f., $275^{604}$, $279,322,383,448^{334}, 452,461^{+448}, 468$, $484,485^{+668}$

Karl IV., König von Frankreich 49, 140f., $178^{196}, 283,429$

Karl I., König von Neapel 244

Karl II., König von Neapel 244

Karl I. Martell, König von Ungarn 447

Karl von Navarra 461

Karl, Herzog von Durazzo $272^{594}, 310$

Karl Martell, Sohn Johannas I. 246, 278

Kasimir, König von Polen 467

Katharina von Siena, Heilige $491^{726}$

Klara von Assisi, Heilige $486^{686}$

Klicman, Ladislaus 4

Konrad von Megenberg $35^{88}, 113^{139}$

Leclercq, Henri 1

Lenzenweger, Josef 4

Lionel, Sohn Edwards III. $148^{76}$

Louis d'Espagne $171^{164}$

Louis de Poitiers $171^{164}$

Louis Sanctus de Beeringen $457^{420}$, $495^{755}$

Luca Manelli 67

Luca Savelli 284

Lucas, Elekt von Lodi 233

Lucas de Flisco, Kardinal $31^{71}, 453$

Ludwig IV., gen. »der Bayer « 52, 232, $240^{+451}, 249 f$., $310,383,432,464$

Ludwig IX., König von Frankreich $140^{+36}$, 244

Ludwig, König von Ungarn 52, 246, 271, $272^{+592}, 279,286,292,316,319,460,468$, $469^{523}$

Ludwig von Aragon 257

Ludwig de la Cerda, gen. »Ludwig von Spanien $\ll 383$

Ludwig von Duras, Titularkaiser von Konstantinopel 318

Ludwig, Herzog von Tarent $274^{600}, 282$, 301f., 308, 311

Lukrez $291^{663}$

Luther, Martin $117^{153}, 215^{347}$

Margarete von Luxemburg, Königin von Ungarn 310

Marguerite Bertrand, Mutter von Kardinal Pierre Bertrand jun. 483

Marguerite de l'Isle $472^{+550}$

Maria, Gottesmutter 125

Maria von Flandern, Mutter von Kardinal Gui de Boulogne 460
Maria von Neapel, Schwester Johannas I. 271

Marie de Cambon, Mutter von Kardinal Pierre Roger de Beaufort 490

Marsilius von Padua 47

Mathieu Bertrand, Vater von Kardinal Pierre Bertrand sen. 485

Matthäus von Verona $284^{638}, 308$

Mollat, Guillaume 2, 5, 6,72

Mornay, Philippe de 1

Moses 19, 155, 221

Nehemia, Prophet $212^{332}$

Niccolò Capocci, Kardinal 2336, 37, 78 10 , 79f., 93, 476-478

Nicolas de Besse, Kardinal 46, 6179, $77^{6}$, $78^{8}, 79 \mathrm{f}$., $81^{27}, 82 \mathrm{f}$., 91, 94f., 97-99, 102f., $114,120^{161}, 229^{410}, 414^{83}, 471^{534}, 478 f$., $487^{695}$

Nicolas de Lyre 469

Nicolino dei Fieschi 175,184

Nikolaus II., Papst 18

Nikolaus IV., Papst 23

Nikolaus Rosell $475^{581}$

Odo IV., Herzog von Burgund 486

Opicinus de Canistris $213^{340}$

Orsini, Bertold 284

Orsini, Francesco $286^{648}$

Orsini, Giovanni 443

Orsini, Matteo 432, 442

Orsini, Napoleone, Kardinal $32^{76}, 86^{43}$, $470^{528}, 494$

Orsini, Perna 431

Orsini, Raymundus $260^{539}$

Orsini, Rinaldo, Kardinal $23^{36}, 24^{37}, 32^{76}$, $78^{10}, 79 \mathrm{f}$., 93, 494f.

Papencordt, Felix 1

Pasteur de Sarrats, Kardinal $37,78^{10}, 80$, $81^{23}, 82^{31}, 205^{308}, 479-481$

Pastor, Ludwig 1

Paulus, Apostel 222, 433

Paulus Diaconus 291+663

Pedro Gomez, Kardinal 31, 37-39, $120^{163}$, $165^{+137}, 166-168,175,184^{215}, 414^{83}, 441$, $442^{276}, 481 \mathrm{f}$.

Pélissier, Antoine 6

Peter IV., König von Aragon 150, 436, $439,459,465,489^{716}$

Petrarca, Francesco $1,39,41,59,83$, $117^{152}, 134,137,213^{340}, 245,283^{636}$, 
$285^{646}, 305,432^{181}, 442^{281}, 448,457$, $458^{422}, 461,473,490,495^{755}$

Petrus, Apostel 108126, 228, 267, 433

Petrus Comestor 290

Petrus Damiani 18

Petrus Lombardus $83^{31}$

Petrus de Paludis $35^{92}$

Philip de Sanguineto 282

Philip of Weston $192^{249}$

Philipp IV., König von Frankreich, gen. »der Schöne« 140

Philipp V., König von Frankreich 485

Philipp VI., König von Frankreich 37, 45, $50,52^{+45}, 53^{47}, 140,141^{38}, 142-144,148$, $175,177^{191}, 181,184,201^{290}, 203^{+298}, 204$, $206,207^{+314}, 209,229,259,430,441,447$, 452, 464

Philipp von Tarent 466

Philippa, Tochter Edwards III. 186

Philippe de Vitry 62

Philippus de Antilla 251

Pictavin de Montesquieu, Kardinal $78^{10}$, 80f., 482f.

Pierre Ameilh $491^{724}$

Pierre d'Arrabloy, Kardinal 432

Pierre Auriol 47

Pierre Bertrand jun., Kardinal $19,78^{8}, 80$, $81^{27}, 82,91,93-95,97 \mathrm{f} ., 100,120^{161}$, $414^{83}, 430^{166}, 436,438,448^{334}, 483-485$

Pierre Bertrand sen., Kardinal $43^{123}, 91$, $120^{161}, 120^{163}, 137^{23}, 273^{+597}, 310,414^{83}$, $444^{294}, 454^{396}, 484-487$

Pierre des Chappes, Kardinal $94^{+73}$, $390^{28}$

Pierre de Châtelus, Abt von Cluny 428

Pierre de Cros, Kardinal $77^{6}, 7^{10}, 80$, $81^{23}, 102 f$., $229^{410}, 414^{83}, 487 f$.

Pierre de Cugnières $171^{164}$

Pierre Desprez, Kardinal 37, 43 ${ }^{123}, 87^{43}$, $120^{163}, 129^{193}, 133^{+5}, 142,145 f ., 153$, $157^{+105}, 163,165,168,170,175,177 f$., $196,207^{312}, 414^{83}, 444^{+294}, 454^{+396}$, 488-490

Pierre des Essars 149

Pierre Roger de Beaufort, Kardinal (Papst Gregor XI.) 6, 32, 46, 61 $79,77^{6}$, $78,81^{27}, 82,102,106^{117}, 112,114-116$, $119-122,194^{255}, 229^{410}, 323,325^{25}, 414^{83}$, $462,469,471^{534}, 490 f$.

Pierre Thomas $42,61^{79}, 68,72^{130}, 448$

Pierre de Viviers $53^{48}$

Pietro de Miredollo 147

Pietro Stefaneschi 431
Raymond de Canillac, Kardinal $77^{6}, 78^{10}$, $80,81^{26}, 82^{31}, 440,471^{534}, 487^{695}$, $491-493$

Raymond de Farges, Kardinal $23^{36}, 37$, $144,147,165 f$., $167^{+145}, 195^{261}, 261,429$, 454, 493f.

Raymundus Pellegrini $151^{89}, 186,197$, $198^{272}$

Raynaldus de Molinis $391^{24}$

Renouard, Yves 5

Richard Fitz-Ralph 464

Richard Lescot, Chronist $456^{410}$

Richard Sprygonell 184

Riezler, Sigmund 3

Robert Bruce, König von Schottland 453

Robert von Neapel, genannt »der Weise « $52,125^{176}, 244 \mathrm{f}$., $246^{476}, 250^{+491}, 254$, $260^{533}, 271,272^{592}, 278,428,438,459$

Robert, Herzog von Tarent 246, 274600, $277^{613}, 310$

Robert VII., Graf von Boulogne, Vater von Kardinal Gui de Boulogne 460

Roboam, Sohn Salomons 116

Roger Mortimer of Wigmore 140

Rogerius de Platea $137^{21}$

Salomon 116

Samaran, Charles 5

Sancia de' Cabanni 278

Sanudo, Marino 428

Sauerland, Heinrich V. 4

Saul, at. König 293

Schäfer, Karl Heinrich 5

Schmitz, Philibert 70

Seba, Rebell aus dem Stamm Benjamin 293

Seneca $67,116^{149}, 119,268,412^{76}$

Shakespeare, William $196^{264}$

Simon de Buissi $171^{164}$

Stephen de Ketelbergh $452^{370}$

Thomas von Aquin, Heiliger 47, 68, 70121, $383,431,466$

Thomas Jorz, Kardinal $78^{16}$

Thomas Waleys $49,206^{310}, 433$

Thomas Walsingham 228

Timotheus 116, 128, 129

Tobias, at. Gestalt 100

Urban II., Papst 20

Urban V., Papst 6, 426, 462, 466, 479, 490, 492 
Villani, Giovanni, Chronist 247, $304^{714}$

Villani, Matteo, Chronist 39, 59, 304714, $440^{265}, 471$

Vinzenz von Beauvais 291

Visconti, Bernabo 426

Visconti, Galeazzo 443

Visconti, Giovanni $34,51,234^{427}, 272^{594}$, $300,304^{716}, 311,461^{448}, 467$

Visconti, Luchino $234^{427}$

Visconti, Matteo 428, 443
Walter Burley $313^{+756}$

Weber, Max 327

Weiß, Stefan 5

Werner von Ürslingen 294, 301

Wéry de Pomerio, Abt von Stablo 481

Wilhelm von Ockham $35^{92}, 213^{340}$

William Bateman 175, 184

William of Norwich $144,151^{89}$

Wood, Diana 6 


